\title{
Quand la critique académique entre dans l'Institution : réflexions sur la structure du champ des politiques économiques
}

When Academic Critique Gets Inside the Institution: Reflections on the Structure of the Field of Economic Policies

\section{Frédéric Lebaron}

\section{(2) OpenEdition Journals}

\section{Édition électronique}

URL : http://journals.openedition.org/conflits/19623

DOI : $10.4000 /$ conflits. 19623

ISSN : $1777-5345$

Éditeur :

CCLS - Centre d'études sur les conflits lilberté et sécurité, L'Harmattan

Édition imprimée

Date de publication : 31 décembre 2017

Pagination : 45-58

ISBN : 978-2-343-14601-0

ISSN : $1157-996 \mathrm{X}$

\section{Référence électronique}

Frédéric Lebaron, «Quand la critique académique entre dans l'Institution : réflexions sur la structure du champ des politiques économiques », Cultures \& Conflits [En ligne], 108 | hiver 2017, mis en ligne le 31 décembre 2019, consulté le 30 mars 2021. URL : http://journals.openedition.org/conflits/19623 ; DOI : https://doi.org/10.4000/conflits.19623 


\section{Quand la critique académique entre dans I'Institution : réflexions sur la structure du champ des politiques économiques}

\section{Frédéric LEBARON}

Frédéric Lebaron est professeur de sociologie à l'ENS Paris-Saclay, membre de l'IDHES-Cachan. Il travaille sur la sociologie des politiques économiques.

T es politiques économiques constituent un champ de lutte et de forces au Lein duquel divers agents sont situés dans des relations objectives de concurrence afin d'imposer une certaine définition de ces politiques. Ces relations sont constamment agitées de mouvements et de tensions potentielles, dans la mesure où les politiques économiques s'ajustent en permanence à un environnement changeant.

Alors que les approches économiques ont souvent tendance à réifier les acteurs institutionnels (gouvernement, banque centrale, etc.), la perspective de sociologie économique relationnelle présentée ici ${ }^{1}$ consiste à « descendre » au niveau des acteurs individuels et de la distribution des multiples espèces de capitaux qu'ils peuvent mobiliser dans des contextes divers pour tenter d'imposer des « règles du jeu » particulières, tout en les situant en permanence dans un espace plus large, au sein duquel les institutions « agissent » également en tant que telles.

L'exemple de la zone euro permet ainsi de mieux illustrer la complexité multidimensionnelle et « multi-niveau » (ou " multi-échelle ») de cet espace de luttes, au sein duquel des acteurs très divers tentent d'infléchir la définition-même $\mathrm{du}$ « cadre » de la politique économique, et celle des politiques concrètes qui y sont menées. Celles-ci vont des plus juridiquement cristallisées (l’indépendance de la Banque centrale, ou le contrôle et la surveillance des

1. Cette réflexion s'appuie en premier lieu sur Bourdieu P., Les structures sociales de l'économie, Paris, Seuil, 2000, et plus largement sur les développements contemporains de la sociologie économique. Voir Steiner P., La sociologie économique, Paris, La Découverte, 2011. 
budgets nationaux, par exemple), jusqu'au plus étroitement dépendantes des contextes et d'arbitrages quasi-quotidiens (la politique de prêts et de taux de cette même banque, par exemple).

Dans ce champ, on peut, de façon provisoire et schématique, distinguer deux « niveaux » ou, mieux, deux structures emboîtées : le champ des politiques économiques au sens le plus large peut être défini comme l'espace, potentiellement très vaste, constitué par le surgissement d'un enjeu particulier, qui mobilise toutes sortes d'acteurs, de tailles et de natures extrêmement diverses : entreprises, groupes de pression, syndicats, partis, etc. Le champ au sens le plus strict, lié au processus historique de formation des États et de division du travail politique, est celui qui est constitué par les relations propres aux professionnels de l'action publique (politiques, administratifs, conseillers et experts) impliqués dans les institutions clés de la politique économique : ministères de l'économie et des finances, banques centrales et, en Europe, Conseil européen, Commission européenne, etc. Ce champ (ou sous-champ) relativement autonome est au cœur de la «fabrique » quotidienne des politiques économiques : production et application du cadre normatif et institutionnel, mesures ordinaires de gestion macro et microéconomiques.

À travers l'exemple de l'arrivée au pouvoir de Syriza (gauche radicale) et la nomination au poste de ministre grec des finances de Yanis Varoufakis, en janvier 2015, on peut observer de façon en quelque sorte « grossie » la tension entre ces deux structures emboîtées constitutives du champ des politiques économiques : dans ce cas exemplaire, en effet, un acteur issu du champ élargi, économiste académique hétérodoxe de notoriété internationale ${ }^{2}$, entre avec une visée de transformation dans le champ en sa définition la plus stricte : tout se passe alors comme si un «corps étranger » suscitait un ensemble de réactions successives conduisant à son rejet. Ces divers processus sont, dès lors, révélateurs des logiques ordinaires de fonctionnement du champ, dans une période où se reconfigure une orthodoxie institutionnelle dont l'efficacité est mise en cause par la crise de la zone euro.

\section{Une double structure}

Le champ des politiques économiques européennes ainsi conçu peut donc être décrit à travers les deux structures qui interagissent : ce que l'on peut donc appeler champ restreint, constitué par l'ensemble des agents directement efficients, à un moment donné, en matière de politique ou « gouvernance » éco-

2. Les «biographies » de Yanis Varoufakis mériteraient en elles-mêmes un article, tant elles ont été constituées en enjeu politique et médiatique durant son bref passage au cœur du gouvernement grec et des institutions de la zone euro. Voir également son texte le plus autobiographique : Varoufakis Y., Conversations entre adultes. Dans les coulisses secrètes de l'Europe, Paris, Les Liens qui Libèrent, 2017. Cet ouvrage fournit de nombreuses données récentes, à l'appui des analyses présentées ici. 
nomique (membres du Conseil des gouverneurs de la BCE, ministres des Finances, en particulier les membres du Conseil européen et de l'Eurogroupe, très hauts fonctionnaires des directions économiques de la Commission, etc.) ${ }^{3}$, et le champ élargi, qui inclut l'ensemble des agents qui prennent position sur la zone euro, sa viabilité, son avenir, etc., et dont certains peuvent le cas échéant aspirer à intégrer le champ restreint tout en menant principalement leurs activités au sein d'autres espaces sociaux (finance, université, politique, etc.).

Cette double structure nécessite de garder présent à l'esprit le fait que les rapports de force internes au champ dans son acception restreinte sont situés dans une relation d'homologie structurale et d'interdépendance avec ceux qui affectent plus largement le champ élargi des politiques économiques, dont la structure mériterait une investigation à elle seule.

Étudier les politiques économiques suppose donc de mener une double approche relationnelle : une, très globale (qui ne sera pas développée ici), porte sur l'ensemble des agents qui tentent d'une manière ou d'une autre de « peser » (selon une métaphore qui rend compte de leur «visée » d'efficacité) sur les mesures adoptées et le cadre général, et une autre, plus spécifique, qui s'intéresse à la logique interne du champ restreint voué à la gouvernance publique de l'économie.

L'espace des prises de position, révélateur de l'état des rapports de force au sein du champ des luttes sur l'euro et la zone euro

Prendre position suppose qu'un « problème public ", ou au moins un « enjeu », soit préalablement construit, suscitant précisément un ensemble de discours spécifiques. Dans le cas de la crise de la zone euro, c'est le cas à partir de la fin 2009, lorsque la spéculation avivée sur la dette publique grecque soulève un enjeu plus global pour l'avenir de la monnaie unique ${ }^{4}$. L'accroissement des écarts entre les taux d'intérêt (spread) sur les titres des dettes souveraines s'accompagne alors de divergences importantes des niveaux d'endettement public, en particulier à la suite de la crise des subprimes et des politiques menées pour y faire face. Ce contexte « macroéconomique » particulier justifie à la fois une mobilisation discursive et institutionnelle portant sur le cas grec, mais aussi, plus largement, des inquiétudes sur la soutenabilité des dettes souveraines et, dès lors, sur l'avenir même de la zone euro, jusque dans ses fondements juridico-institutionnels.

3. On parle ici de "sous-champ de la gouvernance économique ", situé à l'intérieur du champ de la gouvernance européenne.

4. Ces enjeux ont été abondamment décrits par ailleurs. Par exemple, Lebaron F., « La BCE : flexible dans la stabilité ? ", Économie et institutions [En ligne], $\mathrm{n}^{\circ} 22,2015$, mis en ligne le $1^{\text {er }}$ juin 2015. 
L'émergence d'un espace politico-discursif centré sur l'avenir (économico-institutionnel) de la zone euro n'est bien sûr en réalité que la transformation, faisant suite à la crise des subprimes d'un espace préexistant, qui s'est en particulier organisé lors des négociations sur l'union économique et monétaire (UEM) : l'une des difficultés est qu'en fait tout secteur de politique publique peut être décrit comme un champ de luttes politico-discursives permanentes, celles-ci évoluant au fil du temps en fonction de nombreux facteurs, et que dans ce champ, des raisonnements, arguments, analyses plus ou moins sophistiqués ne cessent d'être mobilisés et de se confronter.

Dans le cas qui nous occupe, le champ élargi (défini par l'enjeu de politique publique) se structure autour de prises de position que l'on peut qualifier d'orthodoxes au sens strict (elles se fondent en premier lieu sur le droit, c'està-dire plus précisément ici, sur les traités européens, dérivant en l'occurrence du traité de Maastricht), qui présentent des variantes internes, de prises de position hostiles à l'euro (dont le rôle structurant, en dépit de leur caractère minoritaire, n'est pas à négliger), et enfin de tout un ensemble de prises de position « intermédiaires ", qui visent à modifier ou à refonder institutionnellement le cadre de la zone euro afin de le rendre viable, en se situant soit « dans le cadre » soit « hors du cadre » des traités. Les positions auxquelles sont associées ces prises de position renvoient aux structures du champ politique (opposition partis de gouvernement / partis dits «populistes »), à celles du champ économique (avec des entreprises plus ou moins dépendantes du marché européen) et à celles de l'espace social en général.

Le champ restreint, sur lequel nous nous concentrons, correspond à première vue dans sa totalité à une prise de position relativement cohérente et homogène, que l'on peut synthétiser sous le terme d'orthodoxie institutionnelle : en 2010, la recherche d'un "retour à la normale » s'est traduite par l'adhésion à un scénario de retour au pacte de stabilité et de croissance qui régit le fonctionnement juridico-institutionnel de la zone euro depuis l'avènement de l'euro et donc l'entrée en vigueur de la monnaie unique dans le cadre du traité de Maastricht. Une partie des acteurs du champ restreint insiste toutefois pour que ce rétablissement soit le plus rapide possible, là où d'autres considèrent que le respect des règles est moins urgent, créant de fait une tension latente entre un pôle d'orthodoxie stricte et un pôle d'orthodoxie plus «pragmatique».

Dans le détail, il s'agit donc d'un espace lui-même relativement différencié, mais cette différenciation ne s'est pas traduite par l'apparition de prises de position fortement déviantes jusqu'à aujourd'hui. L'homogénéité des prises de position à l'intérieur d'un champ (fût-il restreint) est, il est vrai, en ellemême signifiante. L'alignement derrière une orthodoxie institutionnelle est une particularité centrale de la construction économique européenne. 
Durant la période de la crise, les caractéristiques des acteurs situés au sein du champ restreint ont peu évolué. On ne voit, par exemple, pas d'inflexion notable du côté des membres du Conseil des gouverneurs de la Banque centrale européenne, ni s'agissant de leurs caractéristiques sociales, qui ont fait l'objet de divers travaux, ni s'agissant de leurs prises de position, du moins en ce qui concerne le cadre juridique général de la zone euro ${ }^{5}$. Qu'en est-il plus largement dans le champ restreint?

Pour étendre l'analyse comparative dans le temps, on a étudié de concert, à titre exploratoire, deux sous-ensembles d'acteurs à partir de sources biographiques, constituant l'ensemble des occupants de positions dirigeantes dans le sous-champ de la gouvernance économique européenne. On les a extraites du Trombinoscope des institutions européennes, qui peut être vu comme un indicateur d'appartenance au champ restreint, en ne retenant que le secrétariat général du Conseil, les directions générales « économiques 》 de la Commission (Ecfin, concurrence, budget, fiscalité, commerce, marché intérieur, industrie et entreprises, Eurostat) et la Banque centrale européenne. On a donc délibérément laissé provisoirement de côté les acteurs strictement nationaux (chefs d'exécutif, ministres des finances, etc.) ou non-européens (Fonds Monétaire International), même s'ils font partie du champ restreint.

Les résultats que nous résumons rapidement ici, déjà présentés par ailleurs ${ }^{6}$, fournissent des éléments empiriques permettant de mieux saisir les enjeux du champ restreint au niveau des caractéristiques des acteurs individuels d'une part, sans pour autant perdre de vue le fait que leur autorité est directement liée à leurs caractéristiques positionnelles (présidence de directoire, présidence de la Commission, etc.) et aux institutions dont ils sont les porte-parole et les «pilotes». Une telle analyse aide à interpréter les rapports de pouvoir tels qu'ils se nouent au quotidien (et pas seulement dans les enceintes les plus officielles) au sein du champ restreint, entre des acteurs individuels, des institutions, des pays, etc.

5. Voir: Lebaron F., Dogan A., « Do Central Bankers’ Biographies Matter ? », Sociologica, $n^{\circ} 2$, 2016 (en ligne), http://www.sociologica.mulino.it/doi/10.2383/85290 ; Lebaron F., « La BCE : flexible dans la stabilité ? ", op. cit.

6. Georgakakis D., Lebaron F., « Le champ de la gouvernance économique européenne et les politiques d'austérité (2010-2015) : premières esquisses ", communication au colloque de Strasbourg, "The economic crisis and the reconfiguration of european actors ", 4 novembre 2015 ; Georgakakis D., Lebaron F., " The field of european economic governance and austerity policies (2010-2015). First drafts ", UACES 46 th Annual Conference, London, 5-7 September 2016 ; Lebaron F., « Les élites européennes comme champ(s). Réflexions sur les usages de la prosopographie et de l'analyse géométrique des données à partir de trois expériences de recherche collective sur des objets transnationaux ", Cultures $\mathcal{E}$ Conflits, ${ }^{\circ} 102$, 2016, pp. 139-162. 


\section{Encadré : l'enquête prosopographique sur les acteurs du champ restreint}

La base de données analysée compte $n=311$ individus. cifique ${ }^{7}$.

On a procédé pour analyser la structure de cet espace à une ACM spé-

On a retenu comme variables actives de l'analyse (entre parenthèses le nombre de modalités actives) les $Q=14$ variables suivantes:

1/ Propriétés socio-démographiques ( 2 variables, 5 modalités) : sexe (2), âge au moment de la publication de la notice (3 : moins de 45 ans / 4560 ans / 60 ans et plus)

2/ Trajectoire scolaire (8 variables, 19 modalités) : lieu principal de formation (3), lieu du dernier diplôme (3), niveau d'études (3), Economie (2), Droit (2), Gestion (2), Sciences Po-Adm pub (2), Sciences (2)

3/ Carrière (4 variables, 9 modalités) : expérience antérieure dans une administration (2), a été membre de cabinet (3), mobilité sectorielle (2), a occupé une position horizontale (2)

La comparaison des deux dates retenues, 2001-2002 et 2012-2013, fait apparaître quelques variations de part et d'autre du " cœur de la crise », la période 2008-2012 ${ }^{8}$. La proportion de cursus « européens » et non " nationaux » augmente nettement, confirmant un processus d'européanisation souligné depuis longtemps ${ }^{9}$. Ce processus contribue à une autonomisation croissante des enjeux européens, dont la dynamique des politiques d'austérité peut apparaître, à certains égards, comme une illustration particulière, dans le cas plus spécifique de la zone euro qui tend à devenir un univers «à part », coupé du reste du champ du pouvoir global ${ }^{10}$, champ clos de luttes entre acteurs très spécialisés, de plus en plus ésotérique pour les acteurs externes. La proportion de détenteurs de $\mathrm{PhD}$ augmente aussi assez nettement, révélant un processus de scientifisation ou d'académisation ${ }^{11}$, qui contribue à rapprocher les enjeux économiques et juridiques de la construction européenne de ceux du monde académique. Ce deuxième mouvement a lieu sous l'impulsion évidente de l'univers du "central banking », lequel constitue non seulement un " pôle », mais sans aucun doute un modèle d'intégration de la politique et de l'expertise scientifique, d'abord dans le domaine monétaire et financier, mais aussi budgétaire et macroéconomique. Ce rapprochement est cependant peut-être à

7. Le Roux B., Rouanet H., Multiple Correspondence Analysis, Thousand Oaks, Sage, QASS, $163,2010$.

8. Le choix de ces deux dates, qui peut sembler relativement arbitraire, permettait de tester l'existence de changements spécifiques entre «l'avant-crise » et «l'après-crise ».

9. Georgakakis D., de Lassalle M., "Genèse et structure d'un capital institutionnel européen. Les très hauts fonctionnaires de la Commission européenne ", Actes de la recherche en sciences sociales, $\mathrm{n}^{\circ} 166-167,2007$, pp. 38-53.

10. Il est en particulier séparé du champ du pouvoir étatsunien.

11. Mudge S. L., Vauchez A., «Fielding Supranationalism. The European Central Bank as a Field Effect », The Sociological Review Monographs, vol. 64, n² 2, 2016, pp. 146-169. 
double tranchant, car il contribue aussi à donner potentiellement plus de force aux contestations des politiques d'austérité issues du monde académique, comme celle lancée après 2010, et surtout 2012, par des économistes américains reconnus ${ }^{12}$.

Les spécialisations disciplinaires restent relativement stables entre 2001 et 2012, avec une légère augmentation des cursus en lettres et sciences humaines, une baisse en sciences et une stabilité globale du rapport économie-droit, qui reste cependant dominé par l'économie. La double domination disciplinaire qui caractérise la construction européenne depuis son origine se trouve renforcée avec la montée en puissance générique du pôle de la gouvernance économique. Les passages par le secteur privé indiqués dans les biographies augmentent aussi de façon assez nette, en particulier s'agissant des avocats, mais aussi du côté de la banque et de la finance.

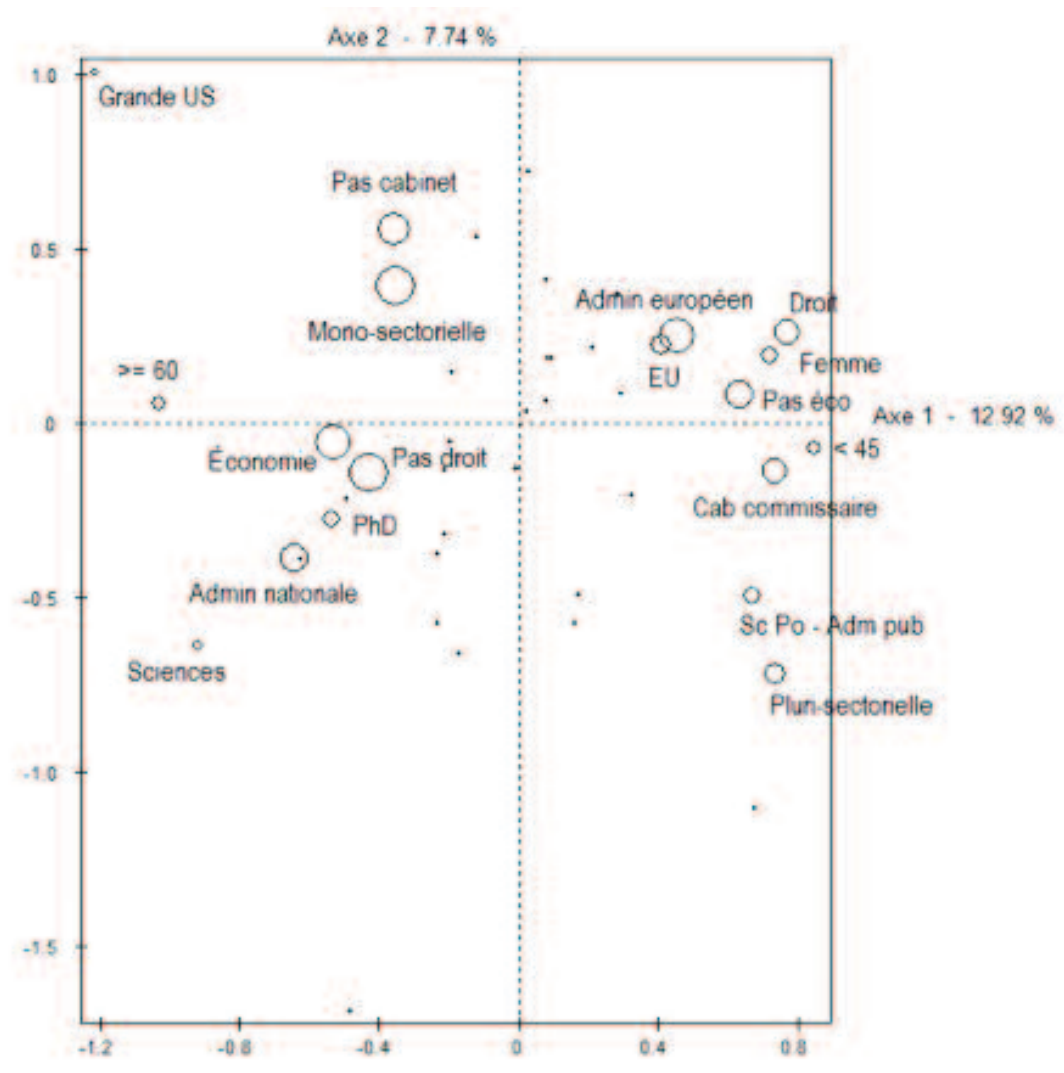

Figure 1a: Plan 1-2. Modalités les plus contributives sur l'axe 1 de l'ACM spécifique

12. Lebaron F., «Zone euro : la crise de la doxa des politiques d'austérité », Idées économiques et sociales, $\mathrm{n}^{\circ} 183$, mars 2016, pp. 39-46. 


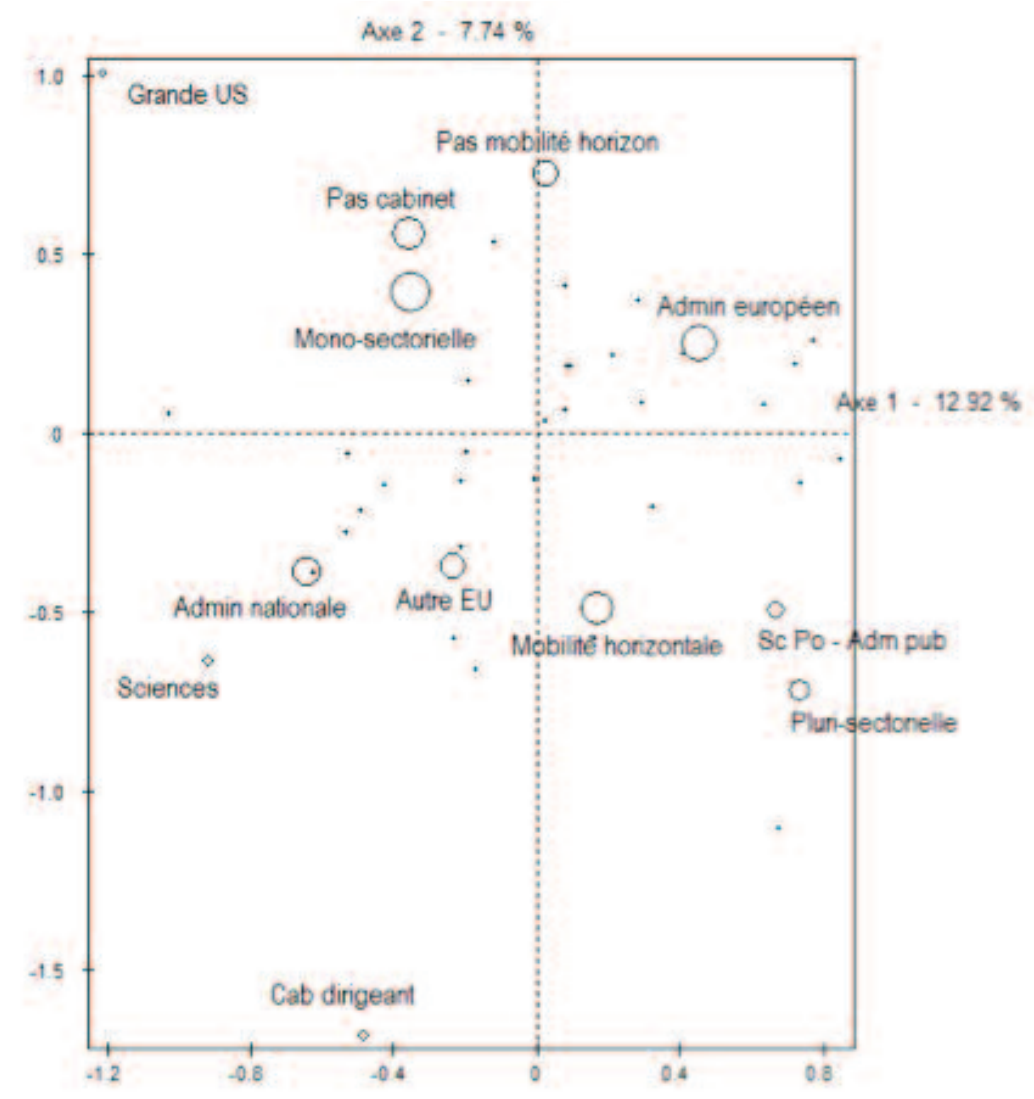

Figure 1b: Plan 1-2. Modalités les plus contributives sur l'axe 2 de l'ACM spécifique

L'axe 1 de l'ACM oppose à gauche (valeurs négatives) : économie, pas droit, administration nationale, et à droite : cabinet de commissaire, droit, pas économie, administration européenne, mobilité pluri-sectorielle, etc. Il définit une opposition entre le capital lié à la science économique, avec une composante américaine, mais aussi associée à l'administration nationale, et le capital juridique, qui apparaît comme plus spécifique des institutions européennes proprement dites («Bruxelles»).

L'axe 2, avant tout déterminé par les questions relatives à la carrière, oppose (en haut) l'absence de mobilité horizontale, de passage par un cabinet, de mobilité sectorielle, l'administration européenne, le passage par une grande université américaine, à (en bas) le passage par un cabinet de dirigeant, la mobilité horizontale et plurisectorielle, l'administration nationale et les 
sciences, les sciences politiques. La surface positionnelle augmente lorsque l'on descend le long de l'axe. Ces différentes dimensions correspondent à des oppositions moyennes entre organisations, ainsi que le montre la figure 2.

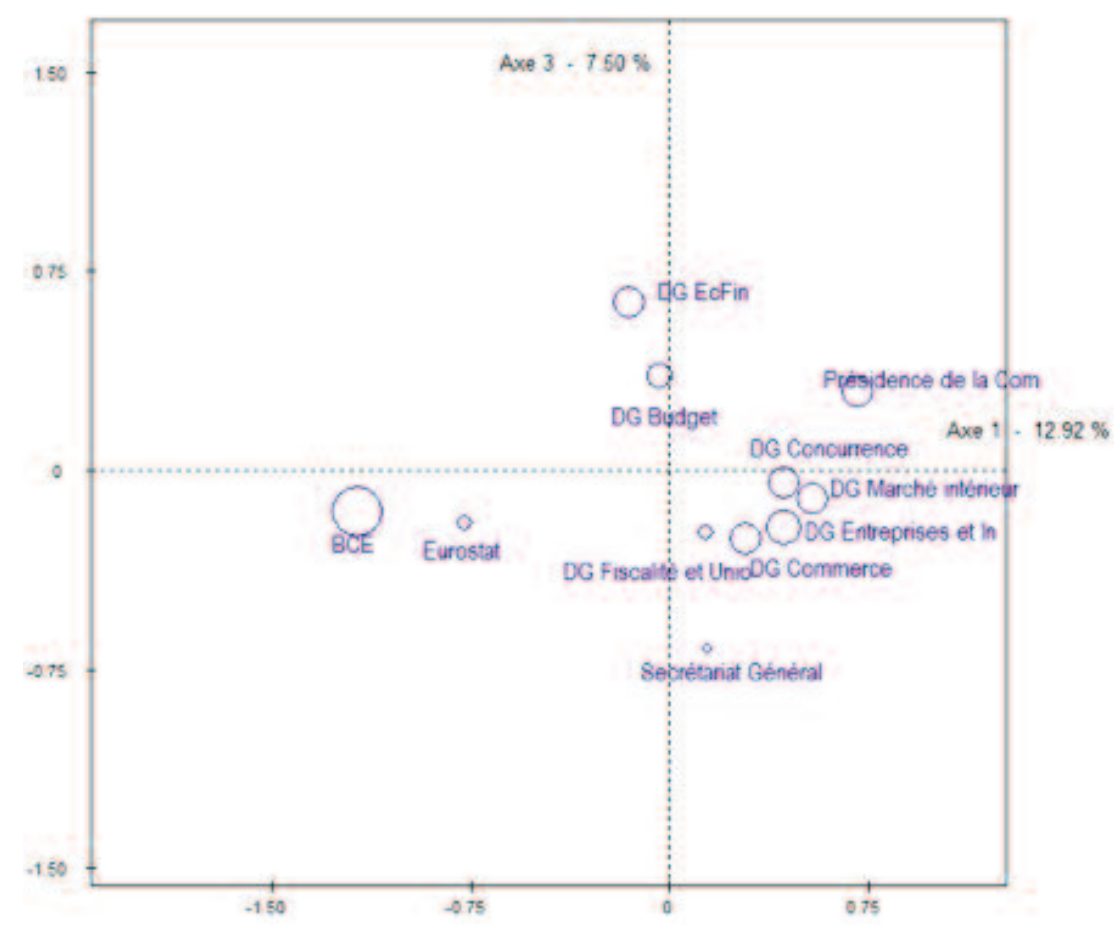

Figure 2 : L'espace des institutions (variables supplémentaires). Plan 1-2.

Une dynamique : éléments d'interprétation

Réactivé dès l'été 2009 par certains dirigeants (allemands) de la BCE, l'enjeu de la réduction des déficits publics afin de combattre l'endettement public massif devient assez rapidement un impératif pour l'ensemble du champ restreint.

$\mathrm{La} \mathrm{BCE}$ donne une impulsion « doctrinale » sur le plan économico-financier, avec les prises de position des banquiers centraux allemands, comme Jürgen Stark, relayées ensuite par le Conseil des gouverneurs. La Commission, à travers la présidence en premier lieu, impulse ensuite une 
«procéduralisation » juridique accrue du retour à l'équilibre, qui va être également portée et légitimée par les acteurs politiques centraux. Ceux-ci sont dominés par les ministres des Finances, d'autant plus puissants qu'ils imposent sur l'agenda l'enjeu de la réduction de la dette publique. L'ensemble du champ s'unifie dès lors autour d'un mouvement global.

Dans ce processus, la dynamique d'un champ de forces caractérisé par des équilibres relativement stables est sans aucun doute plus directement « explicative » que des facteurs "idéologiques » conçus comme sources autonomes issues des acteurs : l'impulsion doctrinale issue de la BCE réactive des dispositions déjà présentes, qui vont ensuite se cristalliser dans des mouvements souterrains d'alignement économico-juridique, lesquels relèvent plus du business (bureaucratique) as usual que d'une croisade austéritaire consciente et structurée. Les acteurs restent insérés dans l'ordre symbolique néolibéral qui définit un « cadre idéologique » global.

L'évolution de la BCE vers le pôle « ouest » de l'espace défini par les deux premiers axes, le plus académique et économique, est illustrée par les différences de position entre Trichet et Draghi. Ce déplacement est associé à une politique de moins en moins « conventionnelle », voire de plus en plus hérétique sur le plan monétaire, qui suscite de très fortes résistances internes et a pour contrepartie un durcissement budgétaire et «structurel».

L'analyse fait apparaître les composantes institutionnelles et individuelles qui sont entrées en action dans le processus d'alignement autour du retour aux politiques d'austérité.

\section{Structures et événements}

Une fois la dynamique structurale ainsi établie, il est possible de comprendre comment une succession d'événements vont contribuer à sa reproduction dans le temps et, le cas échéant, à sa transformation.

La crise de la dette s'accompagne de l'intervention de la «troïka » et la mise en œuvre de politiques d'austérité dures, accompagnées de réformes structurelles ; le cadre juridico-institutionnel est simultanément renforcé dans l'ensemble de la zone euro, avec tout une série de mesures, aboutissant à un nouveau traité, visant à durcir les conditions d'applications des règles juridiques.

Les « événements » qui surviennent durant la période trouvent leur origine principale dans les effets sociaux et politiques des dynamiques économiques à l'œuvre : en Grèce, la hausse vertigineuse du chômage qui accompagne une récession beaucoup plus forte qu'anticipée, se traduit aussi par des 
mouvements de grande amplitude dans le champ politique. C'est la poussée d'une extrême-droite "populiste ", comme dans de nombreux pays européens, mais surtout la montée en puissance des forces sociales critiques, incarnées dans l'espace politique par le parti Syriza.

La contestation intellectuelle se diffuse dans le champ élargi, des ÉtatsUnis jusqu'aux différents pays européens. Elle est notamment incarnée par des économistes critiques comme Yanis Varoufakis et James K. Galbraith qui développent des "propositions » de réforme détaillée de la zone euro, dès $2011^{13}$. Cette mobilisation intellectuelle se traduit par un rapprochement avec des partis politiques contestataires après le discrédit du Pasok, le parti socialdémocrate. Yanis Varoufakis devient même une des figures de Syriza, le parti politique "de gauche radicale » issu de l'eurocommunisme, et James K. Galbraith son conseiller proche. En 2015, la coalition de gauche radicale triomphe aux élections législatives, marquant une première grande rupture dans le champ politique européen.

\section{Un « corps étranger » dans le champ restreint ? Le cas de Yanis Varoufakis face aux politiques professionnels}

Alexis Tsipras arrive donc à la tête de l'État grec après la victoire de son parti de gauche radicale Syriza, qui présente un programme de sortie des politiques d'austérité menées en Grèce depuis 2010, à la suite de la mise en œuvre du programme d'aide européenne porté par la «troïka».

Economiste académique ayant mené une grande partie de sa carrière au niveau international, Yanis Varoufakis entre clairement dans le champ restreint lorsqu'il est nommé ministre des Finances du gouvernement d'Alexis Tsipras en janvier 2015. Il illustre ainsi la figure des économistes académiques embrassant des trajectoires politiques, que l'on retrouve très fréquemment dans le monde des banquiers centraux ${ }^{14}$.

Il devient dès lors membre de l'Eurogroupe et se trouve directement impliqué dans les négociations permanentes entre le gouvernement grec et la « troika ». En juillet 2015, il quitte le gouvernement Tsipras sur le constat d'un désaccord avec la position du premier ministre face aux créanciers. Il ne disparaît pas pour autant de la scène publique, lançant un mouvement paneuropéen (DIEM25) et intervenant régulièrement dans les enjeux politiques et économiques nationaux et européens à travers diverses prises de position.

13. Galbraith J. K., Crise grecque, tragédie européenne, Paris, Seuil, 2016.

14. Lebaron F., Dogan A., art. cit. 
Varoufakis est un universitaire de gauche, à la fois très inséré dans la science économique internationale, « hétérodoxe » selon les catégories de classement usuelles dans le monde des économistes français, et très à l'aise au sein des élites grecques et des médias.

Sa thèse à l'université d'Essex, obtenue en 1987, lui ouvre une carrière universitaire, spécialisée notamment dans la théorie des jeux, qu'il mène successivement en Grande-Bretagne, Australie puis en Grèce.

S'il a milité dans une association d'étudiants noirs en Grande-Bretagne, Yanis Varoufakis a surtout bénéficié de l'insertion de sa famille dans les classes supérieures grecques, avec un père chimiste et président des aciéries Halyvourgiki S.A. Revenu en Grèce en 2000 après avoir travaillé en Australie (où il a acquis la nationalité australienne), il a été conseillé du social-démocrate Giorgi Papandréou entre 2004 et 2006. Cela a contribué à l'accumulation de capital politico-médiatique, qui explique le qualificatif d'économiste «star » qui lui sera parfois attribué, en lien avec son mariage avec l'artiste de renommée mondiale Danae Stratou, et à leurs activités économico-culturelles communes.

La particularité de l'arrivée de Varoufakis à la position de ministre des Finances grec d'un gouvernement de "gauche radicale » est qu'elle a été précédée d'une montée en visibilité régulière depuis l'avènement de la crise grecque, notamment à travers la diffusion de propositions de réformes concernant la zone euro. En développant ce qu'ils appellent leur « modeste proposition ", Varoufakis et son ami James K. Galbraith, fils de John Kenneth Galbraith et professeur d'économie à l'université du Texas, ont pris position au cœur des débats de la zone euro, s'installant dans une position originale entre les contraintes propres au champ restreint (ils s'appuient sur les institutions existantes et ne souhaitent pas engager de refonte des traités) et le champ élargi (leur proposition visant à permettre de sortir de la crise des dettes publiques en relançant l'investissement public à l'échelle européenne).

\section{Le duel Varoufakis / Schäuble comme révélateur}

Comme le montre James K. Galbraith dans le recueil de textes qu'il a consacré à la crise grecque ${ }^{15}$, l'arrivée de Yanis Varoufakis, bien que préparée depuis plusieurs années de familiarisation avec les enjeux internes au champ restreint, est un «choc » institutionnel et politique. Ce «choc» se manifeste tout particulièrement par la confrontation Varoufakis / Schäuble au sein de l'Eurogroupe, qui exprime la tension entre un ontsider porteur d'un projet de 
réforme (aussi "modeste » soit-il) et un insider gardien du cadre des traités, incarnation de l'« intransigeance allemande », selon une expression fréquemment utilisée.

Wolfgang Schäuble est avant tout un homme politique professionnel : il a siégé durant plusieurs décennies au Bundestag pour la CDU, est devenu ministre de l'Intérieur en 1989 et a participé à la mise en place du projet de zone euro au sein du gouvernement allemand dirigé par Helmut Kohl. Si sa carrière connaît une inflexion en 2000 lors du «scandale des caisses noires » qui l'empêche de briguer la chancellerie, il continue d'occuper au sein de la CDU une position clé. En 2009, il est devenu ministre des Finances d'Angela Merkel et va devenir le symbole des positions allemandes les plus dures en matière budgétaire.

Docteur en droit, Schäuble a été haut fonctionnaire au sein de l'administration fiscale du Bade-Würtemberg puis avocat. Sa femme, ingénieur et économiste, a présidé une ONG luttant contre la faim dans le monde. Son expertise économique est celle d'un praticien et d'un juriste de formation, avec un très fort ancrage politique.

Le passage de Y. Varoufakis au ministère des finances, où il s'entoure notamment de J. K. Galbraith, est rythmé par les tentatives de négociation de "sauvetage financier » et de réforme de plus en plus difficiles face aux positions de fermeture opposées par l'Allemagne et incarnées par Schäuble. Celuici, porteur de l'intérêt des contribuables allemand, oppose aux propositions Galbraith-Varoufakis une intransigeance de plus en plus pressante, qui finit par aboutir à une reddition du gouvernement Tsipras aux conditions de l’Allemagne en juillet 2015, suivie du départ de Varoufakis.

Durant toute cette période, l'une des caractéristiques des négociations entre Varoufakis et Schäuble est que le premier ne cesse de mobiliser la science économique à l'appui de ses propositions institutionnelles et, simultanément, de tenter de s'appuyer sur des acteurs intermédiaires pour isoler Schäuble. Celui-ci, en position de force au sein du gouvernement Merkel et dans le champ politique allemand, où il est très populaire en tant que défenseur du contribuable, parvient au contraire à la marginalisation progressive de Varoufakis, avec l'aide des médias dont les plus centraux le décrivent de plus en plus comme un dangereux déviant anti-conformiste, utilisant pour cela aussi bien l'apparence physique et vestimentaire que de prétendus dérapages verbaux, cela au moment où les négociations sont le plus tendues ${ }^{16}$.

16. La stigmatisation prend le plus souvent la forme («soft») de la description distanciée, comme dans le quotidien Le Monde : http://www.lemonde.fr/europe/article/2015/01/27/varoufakisla-nouvelle-tete-des-finances-grecques_4564549_3214.html 
Les rapports de force qui se jouent au sein de l'Eurogroupe entre janvier et juillet 2015 sont bien sûr avant tout ceux qui s'établissent entre un pays économiquement puissant, mais de plus en plus réticent à s'engager dans des politiques de solidarité européenne, et un petit pays financièrement aux abois, qui ne dispose que d'une très faible autonomie relative.

S'il mobilise des capitaux originaux, Yanis Varoufakis entre donc dans le champ restreint dans des conditions d'efficacité très réduites et fait face à de puissants mécanismes de fermeture des possibles. Nous avons vu que ceux-ci reposaient avant tout sur la reproduction d'un groupe très homogène d'acteurs, définissant les logiques propres à ce que nous avons qualifié de «champ restreint ».

Dans ce contexte, on peut se demander, pour conclure, quelles seraient les conditions pour qu'un changement plus effectif atteigne le cadre institutionnel rigide qui régit aujourd'hui la zone euro. On a vu par ailleurs que la Banque centrale européenne avait été le lieu d'une forme d'assouplissement. C'est la conjonction d'une crise grave et l'arrivée d'acteurs plus « pragmatiques ", notamment du fait de leur proximité au monde académique, qui explique ce mouvement au demeurant ambigu : car l'assouplissement monétaire et financier s'est accompagné, en contrepartie, d'un brutal mouvement de rigidification budgétaire, seulement édulcoré après 2014 du fait de ses conséquences ravageuses pour la conjoncture européenne. 